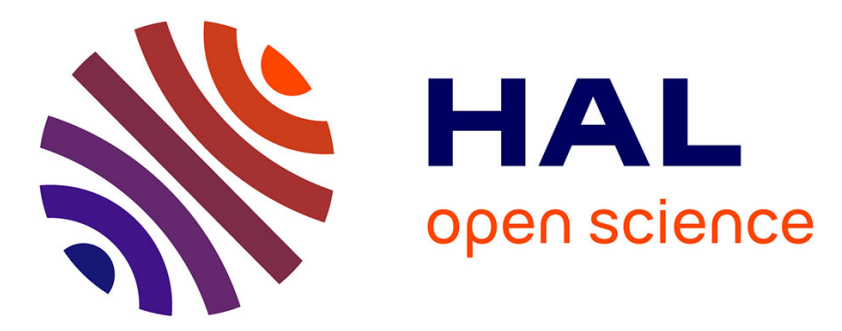

\title{
Designing green network architectures using the ten commandments for a mature ecosystem
}

Nicolas Drouant, Eric Rondeau, Jean-Philippe Georges, Francis Lepage

\section{To cite this version:}

Nicolas Drouant, Eric Rondeau, Jean-Philippe Georges, Francis Lepage. Designing green network architectures using the ten commandments for a mature ecosystem. Computer Communications, 2014, 42, pp.38-46. 10.1016/j.comcom.2014.01.005 . hal-00953000

\section{HAL Id: hal-00953000 \\ https://hal.science/hal-00953000}

Submitted on 1 Mar 2015

HAL is a multi-disciplinary open access archive for the deposit and dissemination of scientific research documents, whether they are published or not. The documents may come from teaching and research institutions in France or abroad, or from public or private research centers.
L'archive ouverte pluridisciplinaire HAL, est destinée au dépôt et à la diffusion de documents scientifiques de niveau recherche, publiés ou non, émanant des établissements d'enseignement et de recherche français ou étrangers, des laboratoires publics ou privés. 


\title{
Designing green network architectures using the ten commandments for a mature ecosystem
}

\author{
Nicolas Drouant ${ }^{\mathrm{a}, \mathrm{b}}$, Éric Rondeau $^{\mathrm{a}, \mathrm{b}}$, Jean-Philippe Georges ${ }^{\mathrm{a}, \mathrm{b}, *}$, Francis Lepage ${ }^{\mathrm{a}, \mathrm{b}}$ \\ ${ }^{a}$ Université de Lorraine, CRAN, UMR 7039, Campus Sciences, BP 70239, Vandoeuvre-lès-Nancy, F-54506, France \\ ${ }^{b}$ CNRS, CRAN, UMR 7039, France
}

\begin{abstract}
The Climate Group notes that energy consumption by ICT activities represented $2 \%$ of global human activity in 2009 [1]. ICT is therefore a sector contributing both to natural resource depletion and to greenhouse gas emission. Moreover, ICT generates considerable quantities of electronic waste, it emits radio waves (including GSM and WiFi) that can adversely affect human and animal health, and it can have an impact on the natural beauty of the landscape (e.g. antennas). A new challenge for ICT engineers is therefore to be able to design ICT more efficiently by considering environmental constraints. In the ecology domain, J. M. Benyus' book "Biomimicry" 2] proposed ten commandments for mature ecosystems. The objective of this paper is to apply these commandments to ICT, by focusing on green network architecture design. The main developments in this research are the proposed relationships between the ten commandments and the networking domain and metrics for assessing both the project-system and the system-of-interest when designing green network architectures.
\end{abstract}

Keywords: Green Networking, Network Architecture Designing, System Engineering, Biomimicry, Mature Ecosystems

\section{Introduction}

Included among future world challenges is the impact of technological development and new emerging technologies on the environment. It will require an enormous effort to address air, water, food, and energy challenges efficiently. The Smart 2020 report [1] written by the international Climate Group recommends the intensive deployment of Information and Communication Technologies (ICT) to enhance the monitoring of the environment and human activities (e.g. industry, building, and transport) and of distributed smart ICT systems that can address pollution, waste, food quality and supply, energy constraints, etc. Therefore, traditional methodologies for distributed system engineering have to be adapted and reviewed to minimize their ICT footprint on the environment in terms of carbon emission, radio wave propagation, waste, technological pollution, energy efficiency, resource reuse, etc. Networked system engineering has to be globally rethought, and [3] has analyzed the relevant literature for energy efficiency support by networks. The contributions of this paper are within the framework of a new ecology paradigm developed in J. M. Benyus' book "Biomimicry" 2]. In the final chapter of this book, "How

\footnotetext{
* Corresponding author

Email addresses: nicolas.drouant@univ-lorraine.fr (Nicolas Drouant), eric.rondeau@univ-lorraine.fr (Éric Rondeau), jean-philippe.georges@univ-lorraine.fr (Jean-Philippe Georges), francis.lepage@univ-lorraine.fr (Francis Lepage)
}

will we conduct business", Benyus defines ten commandments for mature ecosystems. An ecosystem [4] is a complex of living organisms, their physical environment, and all their interrelationships within in a particular unit of space. The evolution of ecosystems generally occurs in two phases: the developing stage and the mature stage [5]. The developing stage involves few species and short food chains. This ecosystem is unstable but highly productive, in the sense that they build up organic matter faster than they break it down. The mature ecosystem is more complex, more diversified, and more stable. Currently, the business model used in our society is the developing stage, and the challenge is to move to the mature stage.

The general objective of this paper is to apply the ten commandments for conducting business to the context of network architecture design. The goal is to check if philosophical concepts from ecology make sense when considering a technical system as a network. To design a technical system, a systemic approach is recommended, which takes into account all requirements and constraints of the global system [6]. In this systemic approach, the design of the system (in this paper, the network architecture) must be evaluated according to two points of view, namely the project-system and the system-of-interest. This paper proposes metrics for both these views. The paper is organized as follows. Section 2 gives the relations between the ten commandments and networked systems, Section 3 defines the metrics for the project-system and in the system-ofinterest, and Section 4 gives a simple example illustrating 
the application of these ideas.

\section{Green network design}

\subsection{Introduction}

The specification of guidelines for designing green network architectures is complex because they must cover different requirement specifications (network, ecology) and must integrate unrelated criteria, criteria that are mutually contradictory, and criteria that are mutually reinforcing. To achieve this issue, this paper proposes to apply system engineering for incorporating the criteria into one model and for analyzing them together during the design process.

System engineering [7, 8] splits the assessment into two parts, namely the project-system and the system-ofinterest parts. The purpose is to be able to use different metrics for evaluating these two parts (Figure 11).

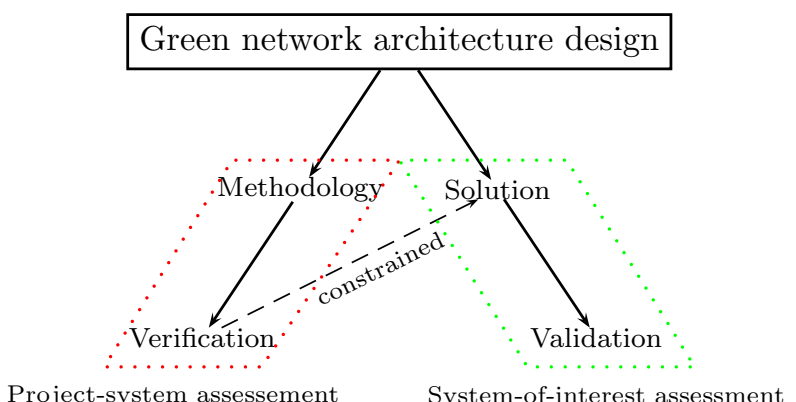

Figure 1: Assessment in system engineering

The objective of the project-system assessment is to define guidelines for designing green network architectures. All its procedures have to be verified to ensure that the methodology of the design has been met by the network designer. Here, the check-list principle is used.

The objective of the system-of-interest assessment is to develop metrics that enable evaluation of different network architecture solutions in terms of the ecology criteria. This is a quantitative evaluation, measured in terms of $\mathrm{CO}_{2}$ emission or the percentage of product recycling, for example. In system engineering, these metrics are called the Measure of Effectiveness (MoE). In general, the MoE will reflect approximately the impact of a given action on the environment.

This paper will consider these two forms of assessment in the field of green networking and will provide to the network engineer both indicators for the project-system and MoEs for the system-of-interest

But the crucial issue is to start by applying ecology concepts to the network domain. Many recent surveys on green networking have collected an impressive number of technical contributions [9, 10, 11, 12, 13], but these have not included the relationship with general ecology concepts developed in [2]. The problem is that, even if a new green network technical solution appears interesting as a local solution, it could have negative effects in terms of the global environmental system. Therefore, the objective is to translate the ten commandments for eco-mature systems into network technical terms, thereby filling the gap between ecology and network domains.

\subsection{Ten commandments}

In the book "Biomimicry" [2], J. M. Benyus says that "over billions of years, natural selection has come up with winning strategies adopted by all complex, mature ecosystems". These strategies are defined as the ten commandments for organisms in mature ecosystems, which

1. use waste as a resource,

2. diversify and cooperate to fully use the habitat,

3. gather and use energy efficiently,

4. optimize rather than maximize,

5. use materials sparingly,

6. don't foul their nests,

7. don't draw down resources,

8. remain in balance with the biosphere,

9. run on information, and

10. shop locally.

The idea is to apply these commandments (or lessons) to economic organization, substituting our current economy by an industrial ecology. In her book [2], Benyus provides examples of the ways the commandments are currently applied in companies or in an economy. The approach developed in this paper is similar, proposing relations between the ten commandments and professional activities in the network domain.

\subsection{Ten commandments for designing network architec- tures}

\subsubsection{Use waste as a resource}

"One of the key lessons from systems ecology is that, as a system puts on more biomass, it needs more recycling loops to keep it from collapsing" [2]. Recycling is a major issue for ICT. The rapid obsolescence of the hardware and software implemented in network devices, which continually offers new functionality with better performance, induces premature equipment renewal and produces electronic waste. During the step of network architecture design, the engineer has to select electronic equipment that can be recycled easily. This recycling has different aspects, including the reuse of old equipment in other applications and equipment dismantling. For example, reuse might involve replacing an old mobile phone with a new one and 
identifying a market for selling the old one to other customers with different needs. In the book "cradle to cradle" [14], the authors propose to design products with their raw materials separated into biological nutrients and technical nutrients. The interest is in avoiding the design of "monstrous hybrids" and in facilitating the recycling step by one part being dedicated to biological metabolisms and another part dedicated to technical metabolisms (enabling the recovery of rare materials, for example). The designer of a network architecture has to reject the electronic "monstrous hybrids", has to anticipate the architecture dismantlement issue, and has to take into account the rate of recycling of electronic components used in the network architecture. The recycling issue concerns not only materials but also energy. Electronic equipment consumes energy and dissipates heat. This heat can be reused as a resource for other applications. For example, the heat emitted by a data centre can provide heat for buildings [15].

\subsubsection{Diversify and cooperate to fully use the habitat}

"In mature ecosystems, cooperation seems to be just as important as competition. Using cooperative strategies, organisms spread out into noncompeting niches and basically clean up every crumb before it even falls off the table" [2]. The idea of this criterion is that the company (in our case an ICT company) has to be aware of the skills offered by other companies and to analyze their complementary and their diversity, thereby improving the competitiveness of all economic actors. Companies have to work "through trade associations, special alliances, and virtual firms to come up with common labelling and materials standards, which will allow them to reuse each other's parts". The communications sector has a central role to federate cooperation between companies by proposing standard communication protocols. One such direction is the deployment of communication systems that implement a new green standard protocol such as IEEE P1888: the Ubiquitous Green Community Control Network Protocol [16]. This standard describes a remote control architecture for digital communities, intelligent building groups, and digital metropolitan networks in terms of the energy, environment, and security domains.

\subsubsection{Gather and use energy efficiently}

"Not everything needed by industry can be recycled, however. Even in a natural system, only nutrients and minerals can be circulated through the diverse connections of an ecosystem; energy cannot" [2]. Through this recommendation, the objective is to install network architectures that use less energy and favour renewable energy sources. The problem of energy consumption in networks has been the focus of the majority of research works in green networking $9,10,11,12,13]$. This is because information equates to energy, with energy use being mandatory for the storage, transport, and visualization of information. In practice, the network architecture designer should select electronic devices with low energy consumption, should optimize the bandwidth (which consumes energy), the network device capacities (number of ports, number of network devices), should develop strategies to manage network sleeping modes, and should monitor network energy consumption (by using the Cisco Energywise technique, for example). In parallel with these network technical considerations, the civil engineering involved in designing buildings or technical rooms hosting the network and the wiring closet should be also taken into account, aiming to optimize the cooling systems.

\subsubsection{Optimize rather maximize}

The general idea of this lesson is to emphasize the quality of the product rather than quantity. Moore's Law, whereby the number of transistors on a chip tends to double every eighteen months, has a direct impact on the network performance requirement. Moreover, from the earliest days of the Internet, the deployment of network architectures has focused on the bandwidth capacity. To increase bandwidth, the communication devices are continually upgraded or replaced, even though they remain functional. New approaches for designing network architectures should be considered that favour the principles of flexibility, reusability, sustainability, and maintenance. The goal is not to sell new network architectures with the best possible performance, but to develop architectures that respect the user requirements. The mechanisms for bandwidth reservation and scheduling, which are usually implemented in networks, are examples of solutions for optimizing communications. The standardization is another way for optimizing energy and raw material. The recent ITU recommendations [17, 18] proposed to specify universal power adapter and charger solutions for both mobile and network devices enabling the consumers to reuse them when they buy new mobile phones, or other electronic equipments. Moreover, Allenby (an American environmental scientist [19]) says, "Our economic system is geared to the sale of many widgets. If we change that to the maintenance of many widgets, we change what we care about". The network business should be aligned with this maintenance model recommended by Allenby.

\subsubsection{Use materials sparingly}

"Organisms build for durability, but they don't overbuild. They fit form to function, building exactly what is needed, with the bare minimum of materials and fuss" [2]. The action envisaged is similar to that recommended for the fourth commandment. The network architecture should be optimized and has to be appropriate for the user requirements. It is not necessary to oversize the network architecture, but to offer only the adequacy of service expected by the customer. The thin provisioning often used to optimize the data storage can be an interesting strategy in the management of bandwidth allocation. The virtualization concept is also a way to reduce the use of materials. Moreover, the concept of functional economy is 
recommended and is based on "leasing as a way of life". In this context, the network companies do not sell network equipment, but instead sell communication services. The interest is that, "since the company would be responsible for uninterrupted service, the products it made would be reliable, heavy duty, and easy to repair and upgrade". The networking business is based mainly on the definition of a Service Level Agreement (SLA), which conforms to the concept of a functional economy.

\subsubsection{Don't foul their nests}

"Organisms must eat, breathe, and sleep right in their manufacturing facility, their habitat; they can't afford to poison themselves" [2]. Network engineers have to design network architectures that mitigate pollution levels. The pollution is not limited to a rate of $\mathrm{CO}_{2}$ emission (and then energy consumption as mentioned in section 2.3.3), but also includes radio waves in wireless architectures and aesthetic issues.

The last point is culture-dependent and cannot be formalized by metrics. Concerning Radiowaves health effects, they was intensively studied by national and international organisms like ICNIRP (International Commission on Non-Ionizing Radiation Protection), IEEE and WHO (World Heath organization). The effects of electromagnetic fields on the human body depend not only on their field level but on their frequency and energy. All studies results claim that the unique non controversial effect of non ionizing EMF is thermal effect [20, 21]. To avoid it all organisms have determine the maximal acceptable values for EMF 22] associated with the frequency range of the RF channel.

Based on these recommendations, the government of each country or state has defined legal maximum level of EMF generated by wireless networks antennas and maximum Specific Absorption Rate (SAR) value. SAR is a measure of the maximum energy absorbed per unit of mass of the head of a person using a mobile phone. These values are sometime lower than the maximum.

It is not disputed that electromagnetic fields above certain levels can trigger biological effects. For example, weak electromagnetic transmitters with a frequency spectrum between 0.1-10 Mhz can affect animal behaviour, particularly the orientation of migrating birds [23]. But biological effect do not means heath hazard. However researches are actively continuing to confirm that low level, long-term exposure to radiofrequency fields could not generate adverse health effects.

A good improvement in wireless communication is to hide antennas into fake chimneys or fake trees. This is a good way to avoid electromagnetic hypersensitivity effect [24] and to minimize aesthetic issues.

Finally, for this commandment, network engineers should consider the network equipment designed by their suppliers. The network equipment should be clean (see the commandment "Use Waste as a resource") and the suppliers should apply just-in-time manufacturing or made-to-order products to avoid overproduction. Finally, the decentralization of production means avoiding "fouling their nests". This point also relates to the commandment "Shop locally".

\subsubsection{Don't draw down resources}

"Organisms in a mature ecosystem live on harvestable interest, not principle" [2]. This leads to two recommendations. It suggests not using non-renewable resources faster than you can develop substitutes and not using renewable resources faster than they can regenerate themselves.

The aim for the network engineer is to optimize the energy consumption of the network architecture during its lifecycle. The energy consumption should include the energy required to manufacture network devices, the energy used to operate the network architecture, and the energy required to dismantle the network. For any of these aspects, the use of renewable energy should be preferred.

\subsubsection{Remain in Balance with the Biosphere}

R. Ayers and L. Ayers [25] write that, unlike Earth's system, which is characterized by closed cycles, the industrial system as a whole is an open one in which nutrients are transformed into waste but are not yet significantly recycled. As for any linear system, this system is inherently unstable and unsustainable. In addition, Benyus says "All we can do is watch for warning signs" [2]. The network research community could provide significant contributions, particularly by the use of wireless network sensors for monitoring the environment [26, 27, 28].

\subsubsection{Run on Information}

"To avoid overshooting, all the firms in an economy have to be keyed into each other and aware of their interactions with the environment, the way organisms are. What we need to establish are feedback links among and within businesses, as well as feedback from the environment to businesses" [2]. Greenpeace provides feedback on environmental efforts by ICT companies, by analyzing their contributions to achieving global greenhouse gas emission reductions of $15 \%$ by 2020. The Leaderboard (CoolIT challenge) is annually updated to track the progress of the largest ICT brands in three key areas. The first area relates to efforts to offer economy-wide technological climate solutions that contribute to global greenhouse gas reductions. The second area relates to initiatives to reduce their own global warming emissions. Finally, it refers to active engagement in political advocacy and support for sciencebased climate and energy policies.

More generally, a network company should set up internal and external audits on its environmental record in implementing suitable action plans. Finally, the methodbased system engineering proposed in this paper is directly relevant to this commandment. It enables the definition of an MoE for analyzing and reducing the impact of network activities on the environment. 


\subsubsection{Shop locally}

Allenby and Cooper [29] say that "Biological communities are, by and large, localized or relatively closely connected in time and space". In the network business, shoplocally networks are currently difficult to envisage because electronic devices are exclusively manufactured in Asia. However, Content Delivery Network (CDN) is an interesting approach enabling to locate and to deliver popular information content as close as possible to users. Moreover, "Shop locally" commandment is also relevant to the context of a functionality economy, in which a service is the keystone of the company. Currently, network providers offer telephone hotline service support that is implemented mainly in low-income countries. The efficiency and quality of this kind of service is often questionable. A relocation of service activities nearer to the consumers should make maintenance operations easier. Another aspect is the geographical location of the network. The storage network implementation has to take into account the proximity of the power sources, which affects the equipment necessary for its delivery and the losses during transportation.

\section{Metrics for designing green network architec- tures}

\subsection{Introduction}

The objective of this paper is to propose a framework for designing environment-friendly network architectures, taking into account the lessons adopted by mature ecosystems. An analysis of these lessons shows that the majority of them could be translated to network system requirements except for the commandment "Remain in balance with the biosphere". This commandment corresponds to a particular network application (monitoring the environment) but is not directly related to the problem of network architecture design. To achieve these requirements, the network engineer needs both indicators for the projectsystem and MoEs for the system-of-interest.

\subsection{Checklist for project-system}

The translation of lessons to the network domain enables the proposal of a general guideline to help network engineers achieve green network architecture design. This guideline is expressed in Table 1, where two possible answers (Yes or No) are used to check the degree to which ecological criteria are considered in the project-system. For example, the activation of Cisco EnergyWise mechanisms will lead to a "Yes" for the questions "Do you select low-energy network equipment?" and "Do you monitor the energy consumed by the network?". The solution that achieves the most "Yes" responses is the one that most respects the environment. This table is not exhaustive and is an initial set of recommendations that should be updated continuously.

\subsection{Green $M o E$ for system-of-interest}

\subsubsection{Introduction}

The design of a network architecture is a process that includes comparison steps between different technical solutions. The goal is to select optimal results with respect to the user requirements. In the context of this paper, one user requirement is that the network architecture should respect the environment and the lessons of mature ecosystems. Therefore, each comparison step requires a defined MoE to guarantee that the network architecture is appropriately green. In this section, a set of MoEs is identified that link to the lessons. Currently, five MoEs are specified. This list is not exhaustive (a metric regarding how manufacturing waste are disposed would have to be also considered) and the equation expressing each MoE can be refined and updated in the future. The main goal of this paper is to demonstrate a general framework for designing green network architectures. The network architecture is analyzed in consideration of two main factors:

- the impact on the environment of the manufacture of network equipment (router, switch, etc.)

- the impact on the environment of each phase of network use.

Table 2 lists the notations that will be used to formulate the MoEs described below.

\subsubsection{MoE on recyclability}

This metric is related to the commandment "Use waste as a resource" and aims at assessing the recycling of an entire network architecture involving many items of electronic equipment. As a first contribution, we propose the following metric:

$$
\Gamma=\frac{\sum_{i \in \overline{X_{r}}} \rho_{i}}{\left|\overline{X_{r}}\right|}, \quad \overline{X_{r}}=\left(X \bigcup X_{S}\right)-X_{r}
$$

This metric involves the set $\overline{X_{r}}$ of equipment that will never be repackaged (for use in another architecture). In the worst case, it corresponds to the total number of items of network equipment used in the architecture, such as routers, switches, firewalls, wires, access points, computers, etc., plus the equipment used to substitute for existing equipment. Items of equipment might be replaced because of failure, upgrades, or even extensions to the architecture. It is important to note here that this list of equipment has to be considered in terms of the whole architecture lifecycle.

The recyclability rate of an item of equipment $\left(\rho_{i}\right)$ ranges from 0 to 1 , where 1 corresponds to $100 \%$ recyclability and means that the item is fully reusable as a resource in other applications.

To optimize this metric, it is necessary to select highly recyclable equipment, to anticipate any repackaging, and to limit the number of items of equipment to be implemented, except for those that are $100 \%$ recyclable $(\Gamma=1)$. 


\begin{tabular}{|c|c|c|}
\hline Mature ecosystem lessons for the project-system in designing a network architecture & Yes & No \\
\hline \multicolumn{3}{|l|}{ 1) Use waste as a resource } \\
\hline \multicolumn{3}{|l|}{ Do you reuse old network equipment in the new network architecture? } \\
\hline \multicolumn{3}{|l|}{ Do you reuse obsolete network equipment in other applications? } \\
\hline \multicolumn{3}{|l|}{ Do you check the recyclability of new network equipment? } \\
\hline \multicolumn{3}{|l|}{ Do you anticipate the dismantlement of the network architecture? } \\
\hline \multirow{2}{*}{\multicolumn{3}{|c|}{$\begin{array}{l}\text { Do you intent to reuse network heat dissipation in other applications? } \\
\text { 2) Diversify and cooperate to fully use the habitat }\end{array}$}} \\
\hline & & \\
\hline \multicolumn{3}{|l|}{$\begin{array}{l}\text { 2) Diversify and cooperate to fully use the habitat } \\
\text { Do you analyze the complementarities and skills offered by companies located near you? }\end{array}$} \\
\hline \multirow{2}{*}{\multicolumn{3}{|c|}{$\begin{array}{l}\text { Do you implement green application standards to facilitate communications between companies, } \\
\text { administrations, and people? } \\
\text { 3) Gather and use enerov efficiently }\end{array}$}} \\
\hline & & \\
\hline \multicolumn{3}{|l|}{$\begin{array}{l}\text { 3) Gatner and use energy emcientiy } \\
\text { Do vou select low-enerov networks? }\end{array}$} \\
\hline \multicolumn{3}{|l|}{ Do you implement green protocols? } \\
\hline \multicolumn{3}{|l|}{ Do you monitor the energy consumed by the network? } \\
\hline \multicolumn{3}{|l|}{ Do you optimize the number of components used in the network architecture? } \\
\hline \multicolumn{3}{|l|}{ Do you optimize the bandwidth allocation? } \\
\hline \multicolumn{3}{|l|}{ Do you design the network architecture in subnetworks to optimize the sleeping mode periods? } \\
\hline \multicolumn{3}{|l|}{ Do you consider the technical rooms and buildings for optimized cooling systems? } \\
\hline \multicolumn{3}{|l|}{ 4) Optimize rather maximize } \\
\hline \multicolumn{3}{|l|}{ Do you select high quality network equipment? } \\
\hline \multicolumn{3}{|l|}{ Do you propose optimized network architectures with respect to the user requirements? } \\
\hline \multicolumn{3}{|l|}{ Do you organize network architectures that could evolve easily to meet new requirements? } \\
\hline \multicolumn{3}{|l|}{ Do you specify maintainable network architectures? } \\
\hline \multirow{2}{*}{\multicolumn{3}{|c|}{$\begin{array}{l}\text { Do you offer efficient maintenance services? } \\
\text { 5) Use materials sparingly }\end{array}$}} \\
\hline & & \\
\hline \multicolumn{3}{|l|}{ Do you propose leasing to your customers? } \\
\hline \multicolumn{3}{|l|}{ Do you make Service Level Agreements with your customers? } \\
\hline Do you consider the commandment "Optimize rather maximize"? & & \\
\hline 6) Don't foul their nests & & \\
\hline Do you take into account the $\mathrm{CO}_{2}$ emitted by the network architecture? & & \\
\hline Do you take into account the radio waves emitted by the network architecture? & & \\
\hline Do you consider aesthetics when implementing the network architecture? & & \\
\hline Do you select suppliers who manufacture clean network equipment? & & \\
\hline Do you select suppliers working with just-in-time methodologies? & & \\
\hline Do you consider the commandment "Shop locally"? & & \\
\hline 7) Don't draw down resources & & \\
\hline Do you select low-energy network equipment? & & \\
\hline Do you select network equipment requiring low energy in its manufacture? & & \\
\hline Do you design network architectures requiring low energy over all their lifecycles? & & \\
\hline Do you prioritize the use of renewable energy? & & \\
\hline 8) Remain in balance with the biosphere & & \\
\hline & & \\
\hline 9) Run on information & & \\
\hline $\begin{array}{l}\text { Do you organize internal and external audits that evaluate the impact of your company on the } \\
\text { environment? }\end{array}$ & & \\
\hline Do you assess the manufacturing process of your equipment vendors (CO2 footprints, waste)? & & \\
\hline Do you use system engineering in designing network architectures? & & \\
\hline 10) Shop locally & & \\
\hline Do you use local markets for the selection of network equipment? & & \\
\hline Do you use local energy to power network architectures? & & \\
\hline Do you propose local maintenance services? & & \\
\hline
\end{tabular}

Table 1: List of indicators for the project-system 
Table 2: Notations

\begin{tabular}{|c|l|}
\hline Symbol & Description \\
\hline$X$ & Set of equipment for the architecture \\
$|X|$ & Number of items of equipment in the architecture \\
$X_{s}$ & Set of substitute equipment \\
$X_{r}$ & Set of repackaged equipment: $X_{r} \in\left(X \cup X_{s}\right)$ \\
$X_{r}$ & Set of used equipment never repackaged: $X_{r}=\left(X \cup X_{S}\right)-X_{r}$ \\
$\rho_{i}$ & Recyclability rate for an item of equipment $i$ \\
$\Gamma$ & Recyclability rate of the whole architecture \\
$E$ & Energy consumed by the whole architecture \\
$E_{m}$ & Energy consumed by the equipment's manufacturing process \\
$E_{u}$ & Energy consumed by the whole architecture during its use phase \\
$P_{u}$ & Power consumption of the whole architecture during its use phase \\
$E_{d}$ & Energy consumed during the dismantling phase of $X_{r}$ \\
$f$ & Factor related to the environment (air, feed efficiency) \\
$h$ & Number of operating hours per year \\
$\delta$ & Power consumption gain (relative to the traffic profile) \\
$\omega_{i}$ & Traffic load for a switch port $i$ \\
$\epsilon_{i}$ & Power consumption of an item of equipment $i$ \\
$\varnothing$ & Power consumption of a switch in the idle state (no traffic) \\
$\sigma$ & Power consumption of a busy switch port (full load) \\
$\Phi$ & $C O_{2}$ emission for the whole architecture \\
$\alpha$ & Multiplicative gain between energy consumed and $C O_{2}$ emission by country \\
$\tau$ & Multiplicative gain caused by energy transportation losses \\
\hline
\end{tabular}

$100 \%$ recyclability is an ideal objective for a circular economy [14], even if it does not mean zero energy consumption (the second MoE in our study).

\subsubsection{MoE on consumption of energy}

This metric refers to both the commandments "Gather and use energy efficiently" and "Don't draw down resources". It measures globally the energy consumption associated with the network architecture. The equation for the energy consumption is:

$$
\begin{aligned}
E & =E_{m}+E_{u}+E_{d} \\
& =E_{m}+\int_{t=0}^{\text {end of lifecycle }} P_{u}(t) d t+E_{d}
\end{aligned}
$$

There are three stages, namely the manufacturing of the network equipment $\left(E_{m}\right)$, the use of the network $\left(E_{u}\right)$, and the dismantling of the network $\left(E_{d}\right)$.

$P_{u}$ represents the power consumption by the network architecture during its use phase. In the literature, many research works propose expressions for modelling the energy consumed by networks. There are two main classes of proposal. One involves high-level modelling, where the interest is in approximating the energy consumption of general network architectures. The other is specific to network technologies and provides precise outcomes. We outline two examples that illustrate these two classes.

Firstly, 30] considers a high-level model without specifying a networking technology. The objective is to develop a management tool for the France Telecom company that offers higher visibility for the current and future consumption of its network. In this context, the following macroscopic model is defined per year:

$$
\int_{t}^{\text {one year }} P_{u}(t) d t=\sum_{i \in X} \epsilon_{i} \times f \times h
$$

Secondly, in [31], the authors propose a more specific equation for estimating the energy consumption of a network architecture based on switched-Ethernet technology. The model for the Energy Efficient Ethernet switch is:

$$
P_{u}=\varnothing+\sigma \sum_{\text {port } i} \min \left(1, \delta \omega_{i}\right)
$$

Here, $\sigma$ takes into account the difference between the power consumption at full load and the traffic-free power consumption divided by the number of ports in the Ethernet switch.

\subsubsection{MoE on pollution}

The estimate of pollution is directly related to the commandment "Don't foul their nests". Pollution has many forms, including $\mathrm{CO}_{2}$ emission, radio wave propagation, and aesthetic issues. In this paper, only the $\mathrm{CO}_{2}$ component is discussed, with Table 3 giving the values to consider for $\alpha$. It shows the matching rate between energy consumption and $\mathrm{CO}_{2}$ emission in electricity production for some European countries. Since an equipment might be manufactured in one country, used in another one and dismantled in a different one, the gain $\alpha$ is related to the 


\begin{tabular}{|c|c|}
\hline Country & $\mathrm{CO}_{2}$ factor \\
\hline Sweden & 0.04 \\
\hline France & 0.09 \\
\hline Finland & 0.24 \\
\hline Italy & 0.59 \\
\hline Germany & 0.6 \\
\hline Ireland & 0.7 \\
\hline Luxembourg & 1.08 \\
\hline
\end{tabular}

Table 3: Factors for $\mathrm{kg}$ of $\mathrm{CO}_{2}$ per $\mathrm{kWh}$

stage of the lifecyle ( $\alpha_{m}$ will hence correspond to the factor during the manufacturing stage). The estimate of $\mathrm{CO}_{2}$ pollution is then obtained by:

$$
\Phi=\Phi_{m}+\Phi_{u}+\Phi_{d}=\alpha_{m} E_{m}+\alpha_{u} E_{u}+\alpha_{d} E_{d}
$$

Moreover, the commandment "Shop locally" recommends the use of energy produced near the network installation, thereby limiting energy-transport losses. To integrate this commandment into the network architecture design, it is necessary to make visible that part of the $\mathrm{CO}_{2}$ emission caused by energy transportation. Therefore, a factor $\tau$ is added to equation [5] giving:

$$
\Phi=\sum_{s=m, u, d} \frac{\alpha_{s}}{\tau_{s}} \times E_{s}
$$

In fact, $\tau$ represents the additional rate for transporting energy. For example, in France the energy lost during transport is $5 \%$ (i.e. $\tau=0.95$ ).

Equation 6 can be extended to include other local sources of energy, such as oil-burning power generators (to power GSM antennas in an emergency) and solar panels installed locally and dedicated to the network. The impact depends on the nature of the energy (electricity, oil), such that the general metric for $\mathrm{CO}_{2}$ emission becomes:

$$
\Phi=\sum_{s=m, u, d} \sum_{\text {energy source i }} \frac{\alpha_{s, i}}{\tau_{s, i}} \times E_{s, i}
$$

where $E_{s, i}$ represents energy-source component $i$ consumed during the stage $s$ (manufacturing, use or dismantling).

The specification of renewable energy in equation 7 accounts for the recommendations for the commandment "Don't draw down resources". The interest in using renewable energy is both indirect (suppressing energy transport losses because it is locally available) and direct (reducing $\mathrm{CO}_{2}$ emissions). Finally, equation 7 is better suited to the recent context of smart microgrids [32] (enabling better integration of renewable energy produced locally in a proximity energy distribution) rather than the more general equation 5 .

\subsubsection{MoE on relevance to user needs}

This metric is related to the commandment "Optimize rather than maximize" for designing optimized networks and to the commandments "Use material sparingly" and "Run on information" for respecting the user requirements. There are therefore two main aspects:
- The network performance in terms of classical Quality of Service criteria such as responsiveness and reliability,

- Its environmental performance, corresponding to the MoEs on pollution and recyclability.

This MoE brings together various items of information, making it difficult to define a global equation that includes all user needs. A radar diagram is proposed to represent both the network performance and the network specifications. Each MoE then corresponds to one axis of the radar. To improve the radar readability, all axes are normalized and zoomed into the interest points.

The global view offered by the radar diagram should make the analysis of network solutions easier, both for the designer and the customer. The best solution is the one where all the MoE results are in the centre of the radar, but the best one relative to the user requirement is the one where the MoE results are closed and beneath the specification line. To achieve this, each MoE should be minimized, implying that the MoE for recyclability has to be inverted. A radar example (Figure 3) is shown in the next section.

\section{Illustration of green MoE interest: a simple case study}

\subsection{Introduction}

As a case study, consider the interconnection of a storage server to a Local Area Network (LAN) via a switched Ethernet. The scope of the study is limited to the switchedEthernet architecture between the LAN and the storage server. The network solution must respect the user requirements in terms of reliability, recyclability, and $\mathrm{CO}_{2}$ emission. In more detail, the customer specifies the reliability as Service Integrity Level (SIL) 2 33], recyclability above $70 \%$ and $\mathrm{CO}_{2}$ emissions less than $500 \mathrm{~kg}$ over the whole network lifecycle. The network performance is here limited to the reliability, but obviously, additional metrics like service capacity and buffer in capacity may be also considered.

The network designer has followed the project-system guidelines and proposes three solutions to the customer. The first (Figure 2(a) comprises one Ethernet switch and two links. The second (Figure 2(b) doubles the number of lines between the storage server and the LAN. The third (Figure 2(c) implements another line.

Clearly, the third architecture is the best in terms of reliability, but it uses the most equipment, which could have a significant impact on the budget and the environment. In contrast, the first architecture is not the most reliable, but optimizes the use of network equipment. However, choosing from among these three solutions is not easy because of the dependence on multiple parameters. Therefore, the MoEs defined in this paper, which provide quantifiable results, can be used to analyze the critical factors in these three proposals. 


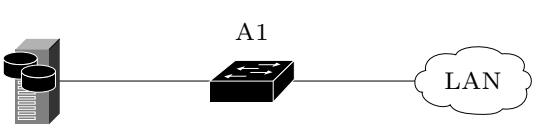

(a) With one line

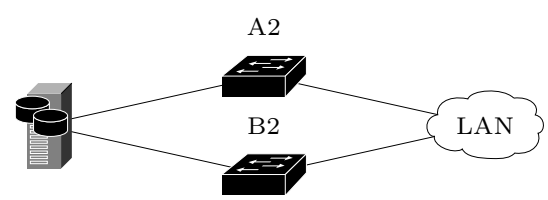

(b) With two lines

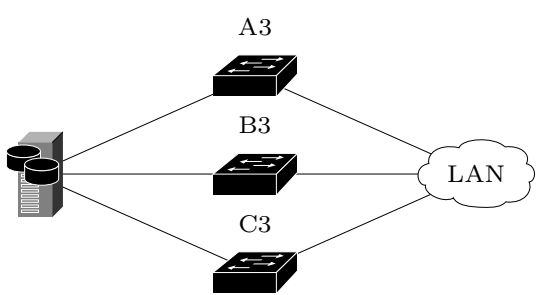

(c) With three lines

Figure 2: Tested scenarii

\subsection{Applying the MoE on recyclability}

It is assumed here that all the Ethernet switch properties are identical, with $\rho_{i}=0.7$, and that all the Ethernet link properties are identical, with $\rho_{i}=0.9$. Moreover, it is planned that, during the usage period of the lifecycle of the network architecture, one switch and one link are dismantled and replaced by new ones. Finally, at the end of the network architecture's life, all the switches are dismantled and all the links are reused in other applications.

Consider now the first architecture. Only two links are repackaged such that $\left|X_{r}\right|=2$. Moreover, Figure 2(a) shows that the architecture comprises three items of equipment (one switch plus two links). Because one switch and one link will be replaced, we have $|X|=3$ and $\left.\mid X_{s}\right]=2$. This gives:

$$
\Gamma=\frac{\sum_{i=1}^{5-2} \rho_{i}}{3+2-2}=76.7 \%
$$

The same approach is then applied to the two other architectures, giving the recyclability results shown in Table 4

The results for the three solutions show that the MoEs for recyclability are similar. In fact, the differences are attributable to the number of switches because all the links are repackaged.

\subsection{Applying the MoE on consumption of energy}

For the network, it is assumed here that the links are 100BaseT, the Spanning Tree Protocol (STP) is activated, and the storage server loads the active line at $10 \mathrm{Mb} / \mathrm{s}$. Moreover, the power consumption of a switch without connected port is $\varnothing=50 \mathrm{~W}$, the power of a connected port with a load of $100 \%$ is $\sigma=15 \mathrm{~W}$, and the power of an idle port is $6 \mathrm{~W}$. With respect to the architecture lifecycle, it is assumed that the usage time for the network architecture is three years, the energy required to manufacture a switch is $750 \mathrm{kWh}$, the energy required to dismantle a switch is $400 \mathrm{kWh}$, the energy required to manufacture a link is $1 \mathrm{kWh}$, and the energy required to dismantle a link is $1 \mathrm{kWh}$.

The estimate of energy used is based on equation (4) with $\delta=18$ (specified as a typical value in 31]). Consider now the first architecture. We first estimate a switch's power consumption during its usage period. For switch A1, it gives:

$$
\begin{aligned}
P_{u} & =\varnothing+\sigma \sum_{\text {port } i} \min \left(1, \delta \omega_{i}\right) \\
& =50+15 \times(2 \times \min (1,18 \times 0.1))=80 \mathrm{~W}
\end{aligned}
$$

where the factor 0.1 corresponds to the utilization rate of the link and the factor 2 to the number of active ports.

The second step includes the manufacturing and the dismantling processes:

$$
\begin{aligned}
E= & E_{m}+\int_{t=0}^{\text {end of lifecycle }} P_{u}(t) d t+E_{d} \\
= & ((1+1) \times 750+(2+1) \times 1)+0.08 \times 24 \times 365 \times 3 \\
& +(2 \times 400+1 \times 1)=4406 \mathrm{kWh}
\end{aligned}
$$

Table 4 gives the results obtained for all three architectures. Compared to Architecture 1, the power consumption for switches B2, B3, and C3 is slightly different:

$$
P_{u}=\varnothing+\sigma \sum_{\text {port } i} \min \left(1, \delta \omega_{i}\right)=50+2 \times 6=62 W
$$

where the factor $2 \times 6$ corresponds to the consumption of ports made idle by the STP.

The results show the costs induced by the energy requirement for manufacturing, supplying, and dismantling the network. In this study, Architecture 3 consumes twice as much as Architecture 1.

\subsection{Applying the $\mathrm{MoE}$ on $\mathrm{CO}_{2}$ emission}

In addition to the previous assumptions, it is assumed that the network architecture is powered only by electricity and that the manufacturing, the dismantling, and the network architecture are implemented in France, for which $\alpha=0.09$.

The $\mathrm{CO}_{2}$ emitted by the network architectures is obtained from equation (7), which gives for Architecture 1:

$$
\Phi=\frac{E}{0.95} \times \alpha=\frac{4406}{0.95} \times 0.09=417 \mathrm{~kg} \text { of } \mathrm{CO}_{2}
$$

The interest in these results is to identify clearly the relationship between the reliability considerations and the 
Table 4: Results for the case study

\begin{tabular}{|c|c|c|c|c|}
\hline Architecture & Recyclability & Energy consumption & $\mathrm{CO}_{2}$ emission & Reliability \\
\hline 1 & $76.7 \%$ & $4406 \mathrm{kWh}$ & $417 \mathrm{~kg}$ & $3 \times 10^{-5}$ \\
2 & $75 \%$ & $7188 \mathrm{kWh}$ & $681 \mathrm{~kg}$ & $9 \times 10^{-10}$ \\
3 & $74 \%$ & $9969 \mathrm{kWh}$ & $944 \mathrm{~kg}$ & $2.7 \times 10^{-14}$ \\
\hline
\end{tabular}

impact on environment. From these values, the network designer has quantitative indicators for guiding the decision process.

The next MoE, on relevance to user needs, enables optimization of the decision according to the initial specifications.

\subsection{Applying the MoE on relevance to user needs}

\subsubsection{Estimation of the MoE on reliability}

In this study, the specification for reliability is to obtain a network with SIL 2 33]. Table 5 provides the values used in the continuous mode. From Table [5, this corresponds to failures per hour below $10^{-6}$.

We define $n_{i}$ as being the number of items of equipment forming a path $i$. Let $\lambda$ be the failure probability per hour for any item and $\mu=1-\lambda$ as the non-failure probability.

\begin{tabular}{|c|c|}
\hline SIL & Range of $\lambda$ (failures per hour) \\
\hline 4 & $10^{-9}<\lambda<10^{-8}$ \\
3 & $10^{-8}<\lambda<10^{-7}$ \\
2 & $10^{-7}<\lambda<10^{-6}$ \\
1 & $10^{-6}<\lambda<10^{-5}$ \\
\hline
\end{tabular}

Table 5: Definition of SIL in continuous mode (IEC 61508)

The next procedure is to estimate the network reliability for the three architectures. It is considered that all switches and links used in this study have failures per hour equal to $\lambda=10^{-5}$. The failure probability of a network $(P)$ composed of $p$ independent paths depends on the failure probability of each path $i\left(P_{i}\right)$ such that:

$$
P=\prod_{i=1}^{p} P_{i}=\prod_{i=1}^{p}\left(1-\mu^{n_{i}}\right)
$$

For example, considering Architecture 3, there are three paths formed by three items (one switch plus two links). This gives:

$$
P=\left(1-\left(1-10^{-5}\right)^{3}\right)^{3}=2.7 \times 10^{-14}
$$

All results are shown in Table 4 .

\subsubsection{Radar diagram for the MoEs}

The radar diagram (Figure 3) collects all the network performances obtained for the various architectures and the performance specification.

The architecture to be selected has to respect all the initial specifications. The radar diagram shows that no

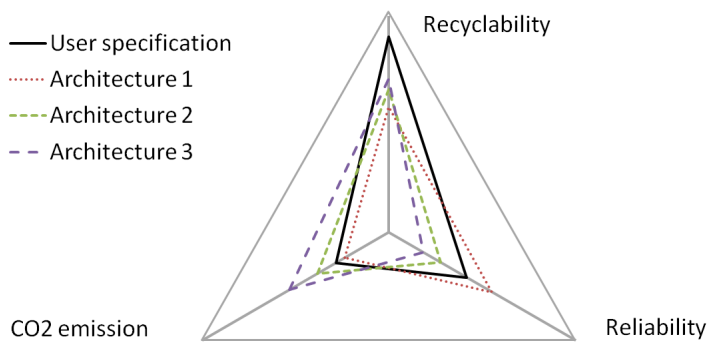

Figure 3: Radar diagram

solution is completely acceptable. Architecture 1 is not reliable enough, and the two other architectures use more network equipment, thereby emitting excessive $\mathrm{CO}_{2}$. The main issue is that the MoEs are interdependent. The radar diagram shows that improving the reliability (from Architecture 1 to 2 and 3) by including network equipment redundancy has an impact both on pollution and recyclability and also on other costs not studied in this paper. Indeed, an economics point of view could be also integrated into the radar diagram to complete it as a tool for designing network architecture.

In conclusion, the radar diagram is a useful tool that enables the identification of appropriate actions that would satisfy the customer. For example, Architecture 1 would be appropriate if the designer selects switches with failures per hour of $10^{-7}$. With respect to Architectures 2 and 3, it is necessary to reconsider the consumption of energy to reduce the $\mathrm{CO}_{2}$ emissions. The network designer could select low-energy switches manufactured by other providers.

\section{Conclusion}

This paper proposes a general methodology for designing green network architectures based on a biomimicry approach. This contribution shows that the philosophical commandments followed by mature ecosystems are directly applicable to a technological context (particularly in the network area) and more generally to a systemengineering approach. These commandments are translated into guidelines for checking the system-project during the conception step and are used for defining metrics (MoEs) that cover environmental constraints. MoEs are necessary for assessing and comparing different network organizations to enable selection of the best solution. A radar diagram is proposed and summarizes the various MoE results regarding the initial specification defined by 
the customer. A major issue is the adjustment of multiple MoEs, which are often strongly correlated and can therefore have opposing effects on the whole system.

A development of this research would be to analyze the correlations between the axes of radar. It would be interesting to provide correlation models that would help the network designer tune the different parameters. Another development would be to integrate this work with the fractal tiles developed for the cradle-to-cradle concept [14]. A fractal tile considers three points of view simultaneously, namely ecology, economics, and ethics. Integrating economics and ethics aspects into the approach proposed in this paper would enlarge the scope of the system. New MoEs should be specified for the economics and ethics aspects (for instance how to choose equipment vendor responsibly). Finally, the MoEs proposed in this paper should be completed and refined to take into account new criteria (such as radio wave pollution), other network technologies, and new green standards for communications.

\section{References}

[1] Smart 2020, http://www.smart2020.org 2012.

[2] J. M. Benyus, Biomimicry: Innovation Inspired by Nature, Harper Perennial, ISBN 0060533226, 2002.

[3] B. Carro, A. Sanchez-Esguevillas, F. Martin, Telecommunications technologies for energy efficiency supported by future networks, IEEE Communications Magazine (2012) 12-15.

[4] Encyclopedia Britannica, http://www.britannica.com/, 2012.

[5] B. R. Allenby, W. E. Cooper, Understanding industrial ecology from a biological systems perspective, Environmental Quality Management 3 (3) (1994) 343-354, ISSN 1520-6483.

[6] A. Pyster, D. Olwell, J. Anthony, S. Enck, N. Hutchison, A. Squires, A Guide to the Systems Engineering Body of Knowledge (SEBoK) version 0.75, Hoboken, NJ: Stevens Institute of Technology, 2011.

[7] S. H. Team, Systems Engineering Handbook, INCOSE, 2010.

[8] G. Morel, H. Panetto, M. Zaremba, F. Mayer, Manufacturing Enterprise Control and Management System Engineering: Paradigms and Open Issues, IFAC Annual Reviews in Control 27 (2) (2003) 199-209.

[9] A. P. Bianzino, J.-L. Rougier, D. Rossi, C. Chaudet, A Survey of Green Networking Research, IEEE Communications Surveys \& Tutorials 2 .

[10] R. Bolla, R. Bruschi, F. Davoli, F. Cucchietti, Energy Efficiency in the Future Internet: A Survey of Existing Approaches and Trends in Energy-Aware Fixed Network Infrastructures, IEEE Communications Surveys and Tutorials 13 (2) (2011) 223-244.

[11] M. Kakemizu, A. Chugo, Approaches to Green Networks, Fujistsu Sci. Tech. 45.

[12] M. Conti, S. Chong, S. Fdida, W. Jia, H. Karl, Y.-D. Lin, P. Mähönen, M. Maier, R. Molva, S. Uhlig, M. Zukerman, Research challenges towards the Future Internet, Comput. Commun. 34 (18) (2011) 2115-2134, ISSN 0140-3664.

[13] E. Mingozzi, X. Perez-Costa, C. Rosenberg, S. Xu, Special issue: Wireless Green Communications and Networking, Computer Communications 35 (14) (2012) 1649-1768.

[14] W. McDonough, M. Braungart, Cradle to Cradle: Remaking the Way We Make Things, North Point Press, 1st edn., ISBN 0865475873, 2002.

[15] Val-Europe project in France, http://www.valeurope-san.fr/info/UK/00, 2012.

[16] Ubiquitous Green Community Control Network Working Group, UGCCNet, IEEE P1888, IEEE Standard for Ubiquitous Green Community Control Network Protocol, 2011.
[17] International Telecommunication Union (ITU), Universal power adapter and charger solution for mobile terminals and other hand-held ICT devices, Recommendation ITU-T L.1000, 2011.

[18] International Telecommunication Union (ITU), External universal power adapter solutions for stationary information and communication technology devices, Recommendation ITU-T L.1001, 2012.

[19] B. R. Allenby, D. J. Richards, The greening of Industrial Ecosystems, National Academy of Engineering, ISBN 0-309-04937-7, 1994.

[20] World Heath Organization (WHO), Electromagnetic fields and public health: mobile phones, Fact sheet $\mathrm{n}^{\circ} 193,2011$.

[21] World Heath Organization (WHO), Base stations and wireless technologies, Fact sheet $\mathrm{n}^{\circ} 304,2006$.

[22] Institute of Electrical and Electronics Engineers, IEEE standard for safety levels with respect to human exposure to radio frequency electromagnetic fields, $3 \mathrm{kHz}$ to $300 \mathrm{GHz}$, IEEE Std C95.1, 2005.

[23] T. Ritz, P. Thalau, J. B. Phillips, R. Wiltschko, W. Wiltschko, Resonance effects indicate a radical-pair mechanism for avian magnetic compass, Nature 429 (6988) (2004) 177-180, ISSN 0028-0836.

[24] H. Seitz, D. Stinner, T. Eikmann, C. Herr, M. Roosli, Electromagnetic hypersensitivity (EHS) and subjective health complaints associated with electromagnetic fields of mobile phone communication - a literature review published between 2000 and 2004, in: Science of the Total Environment, vol. 349, 45$55,2005$.

[25] R. U. Ayers, L. Ayers, Accounting for Resources, Edward Elgar Publishing Ltd, ISBN 18589986400, 1998.

[26] R. Szewczyk, E. Osterweil, J. Polastre, M. Hamilton, A. Mainwaring, D. Estrin, Habitat monitoring with sensor networks, Commun. ACM 47 (6) (2004) 34-40, ISSN 0001-0782.

[27] T. Arici, Y. Altunbasak, Adaptive Sensing for Environment Monitoring Using Wireless Sensor Networks, IEEE WCNC Wireless Communications and Networking Conference 4 (2004) $2347-2352$.

[28] D. Estrin, W. Michener, G. Bonito, Environmental Cyberinfrastructure Needs For Distributed Sensor Network, Tech. Rep., National Science Foundation, 2003.

[29] B. R. Allenby, W. Cooper, Understanding industrial ecology from a biological system perspective, Total quality environmental management 3.

[30] L. S. Foll, TIC et Énergétique : Techniques d'estimation de consommation sur la hauteur, la structure et l'évolution de l'impact des TIC en France., Ph.D. thesis, Institut national des télécommunications, 2008.

[31] P. Revirieg, An Energy Consumption Model for Energy Efficient Ethernet Switches, in: International Conference on High Performance Computing \& Simulation (OPTIM Workshop), Jul., 98-104, 2012.

[32] E. Perea, J. M. Oyarzabal, R. Rodríguez, Definition, evolution, applications and barriers for deployment of microgrids in the energy sector, Elektrotechnik und Informationstechnik 125 (2008) 432-437, ISSN 0932-383X

[33] International Electrotechnical Commission, Functional safety of electrical/electronic/programmable electronic safety-related systems, IEC Standard 61508, 2005. 